\title{
Diffusion of Hydrosilanes from the Control Layer to the Vinylsilane-rich Flow Membrane during the Fabrication of Micro-fluidic Chips
}

\author{
Dong-Jie Guo ${ }^{l}$, Shou-Jun Xiao ${ }^{1}$, Hong-Bo Liu ${ }^{l}$, Jie Chao ${ }^{l}$, Bing Xia ${ }^{l}$, Jing Wang $^{l}$, \\ Jia Pei ${ }^{1}$,Yi Pan ${ }^{1}$, Zhong-Ze Gu ${ }^{2}$, Xiao-Zeng You ${ }^{1}$
}

contribution from ${ }^{1}$ State Key Laboratory of Coordination Chemistry, School of Chemistry and Chemical Engineering, Nanjing University, Nanjing 210093, China.

${ }^{2}$ State Key Laboratory of Molecular and Biomolecular Electronics, Southeast University, Nanjing 210096, China.

E-mail:sjxiao@nju.edu.cn

Calculation of ATR-FTIR peak areas. Since both absorbance peaks ( $\mathrm{Si}-\mathrm{H}$ at 2160 $\mathrm{cm}^{-1}$ and $\mathrm{C}-\mathrm{H}$ at $1260 \mathrm{~cm}^{-1}$ ) are singular and separated from others, a fixed two-point method is used to integrate their peak areas between $2113-2229$ and $1230-1320 \mathrm{~cm}^{-1}$, respectively, with an OPUS 4.2 software.

\section{Mechanical properties.}

Partial stress-strain curves recorded here are to describe generation of the Young's modulus. A typical curve can be classified into two regions: linear (elastic strain) and complex (elastic plus plastic strain) region. The Young's modulus is derived from the 
slope of the linear region in s-Figure 1. Our determination of the Young's modulus varied slightly from batch to batch, due to slight variations in clamping, variations in the density of crack initiation sites on the edges of the samples, variations in the thickness etc. The data fluctuated for $10 \%$. The results presented here represent typical values. For the elongation ratios (at break) and tensile strengths (at break), those data varied significantly from sample to sample. The results fluctuated about $25 \%$. The data given represent the mean values of our determination.

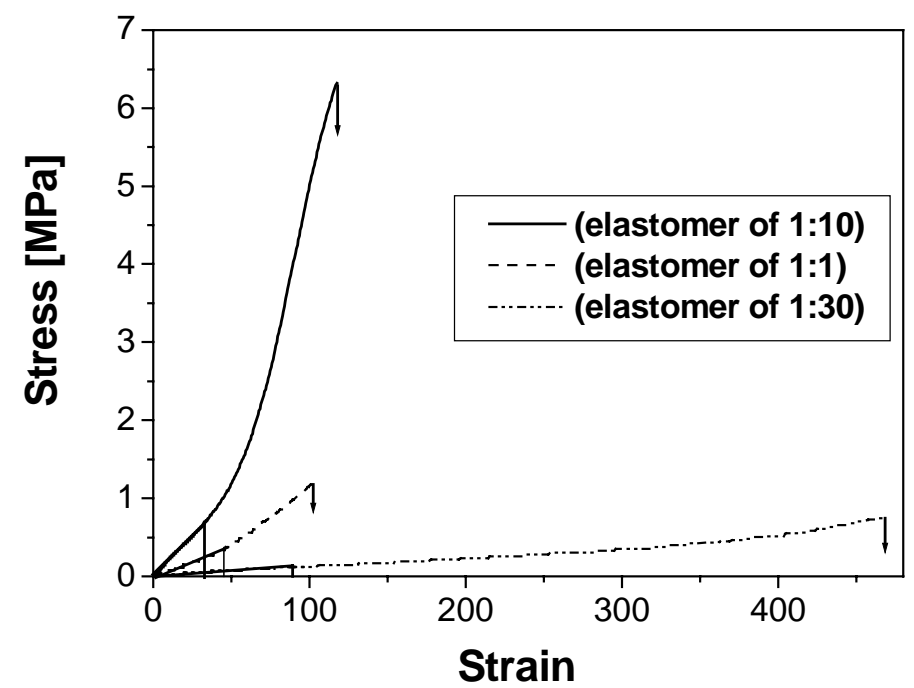

s-Figure 1. The typical stress-strain curves of PDMS networks. The Young's moduli were calculated from the slopes of the curves in the linear (elastic strain) regions indicated by straight triangles. Above the elastic region, the tensile strain is contributed by both elastic and plastic strains. 